\title{
THE
}

\section{JOURNAL OF APPLIED PSYCHOLOGY}

\begin{tabular}{lll}
\hline Vol. II & MARCH, 1918 & No. 1
\end{tabular}

\section{THE ABILITIES OF AN EXPERT MARKSMAN TESTED IN THE PSYCHOLOGICAL LABORATORY}

\section{Arthur I Gates, Teachers College, Columbia Unversity}

The current interest and activity of psychologists in devising and applying tests of special fitness for various vocations required in military operations, may justify a report upon an examination in the psychological laboratory of a man of expert ability as a marksman. Mr. William F. Blasse, of San Francisco, long known as an exceptional marksman, scored the world's record on the 200 yard range, free off-hand shooting, 100 shots consecutively, on September 22, 1916. Using the King Target, Mr. Blasse scored 223 points out of a possible 300 which would have required each of the 100 shots to have been placed within one and one-half inches of the center of the a target. Some idea of the accuracy of Mr. Blasse's shooting 3 is indicated by a detailed score; of the 100 shots, 38 scored - red flags (within 15 mches from the center), 47 blue flags ( 3 inches from center) and 15 white flags ( 6 inches from center). The average distance of the 100 shots from center - would be about 2.3 inches. Thus at a distance of 200 yards, shooting without a rest, Mr. Blasse would find a man's head an easy mark.

Taken into the University of California laboratory, $\mathrm{Mr}$. Blasse gladly submitted to an experimental examination in an effort to determine in what respects his superiority over the marksmen of average or inferior ability was to be found. From many tests tried, only those which possess some special significance are mentioned. As a control group, 10 students in the laboratory consented to go through the same series of tests. 
The group included 2 fair, 4 mediocre, 3 poor marksmen, and one who had shot a gun less than a half dozen times.

First of all it seems important that the expert marksman should have good eye-sight-but does he need to possess exceptional vision? A few stock tests demonstrated at once that while Mr. Blasse's eyes were efficient and without marked defects, they were not markedly above normal. In acuteness of vision, he was not unusual, color vision was normal, neither myopia, hypermetropia nor astıgmatism of noticeable amount was present, and the general muscular control was normal In short, his vision was good but not exceptional

The second group of tests was concerned with the steadiness of motor control. Most of the tests employed are familiar to the psychological laboratory, and I shall economize space by omitting detailed descriptions of apparatus as well as details concerning the data.

The brass plate steadiness test described by Whipple, ${ }^{1}$ with holes whose diameters were $32,20,16,13,11,10$, 9,8 , and 7 sixty-fourths of an inch, respectively, was first used Holding the arm at full length, $\mathrm{Mr}$ Blasse showed considerable superiority to the other 10 subjects, by holding the needle for 3 seconds in the hole of $13 / 64$ inch diameter, while the steadiness of the other subjects failed in the next larger hole (16/64 inch), 6 others touched the sides of the 20/64 inch hole and one student failed in each of the 4 trials in the largest hole (1/2 inch) These relations held approximately for either hand, although the right hand was, on the whole, somewhat more steady

For measures of the steadiness of one form of voluntary muscular control, the "tracing board" test ${ }^{2}$ was employed. Several tests were made with the board placed at different angles, and in all these tests $\mathrm{Mr}$ Blasse was somewhat, but not markedly, superior to the other subjects The most steady student was quite as good.

The so-called "target test," designed to measure the accuracy and precision of movement ${ }^{3}$ consisting in thrusting a pencil at a target placed at arm's length, showed that in this form of motor control, Mr. Blasse was only slightly superior to the average of ten students The three tests just described, when considered together, show that the expert marksman is considerably superior in simple steadiness of motor control, but only slightly superior to the average in accuracy and pre-

\footnotetext{
1Manual of Physical and Mental Tests, 1910, p I24

2 Whipple, $O P$ cit., p I20

3 Whipple, p. II5ff
} 
cision of arm movements. In a final test (the tapping test) which measures mere speed of motor control, Mr. Blasse showed approximately average ability.

Not only is the steadiness of the arm involved in holding a gun, but the steadiness of the body as a whole To measure this form of motor control a simple apparatus was used. A helmet was placed upon the head, projecting upward from the top of which was a stiff pointer. A smooth platform, like that of a punching bag, over which was stretched a paper covered with a thin layer of smoke, was adjusted so that it came into contact with the top of the pointer The subject then attempted to stand motionless, every slight sway of the body being recorded by the tracings of the pointer on the smoked surface. $\mathrm{Mr}$ Blasse showed some superiority in this test, his bodily movements being less marked than those of the steadiest students. The accompanying tracings (Fig. I) show the actual movements made by Mr. Blasse, a student of average, and one of marked unsteadmess An interesting feature brought out by this test was that $\mathrm{Mr}$ Blasse was subject to distraction or suggestion with regard to movements to a much less extent than were most of the other subjects. If I told certain subjects that they were swaying forward, efforts to counteract this imaginary movement would usually result in greater unsteadiness. If I told them to think hard of something to their left, as a rule the body would sway in that direction. A sudden slap of a book on a table usually provoked a series of movements and, most significant of all, if the subject thought intently upon the possibility of his increasing unsteadinessif such ideas as. "I'm awfully unsteady to-day," "There, I moved again," "I can't seem to control myself," were dwelt upon, the results were disastrous. Now, Mr. Biasse was relatively cool and unmoved during all such procedures, although the general surroundings, the apparatus and so on were less familiar to him than to the other subjects, and although a certain fear lurked in his mind that he was not going to do well in these strange tests. The fact that $\mathrm{Mr}$ - Blasse was relatively non-susceptible to motor suggestibility is important

Thus we have found that the expert marksman is possessed of superior steadiness of muscular control in the various stock tests Finally, a test of the steadiness of the gun itself, which would result from various movements in combination, were measured. For one method, a steel needle was fastened to the end of the gun and the movements were recorded by means of the metal plate of small holes mentioned before. In this test, with the gun in hand, Mr. Blasse's superiority was more pro- 
FIG. I

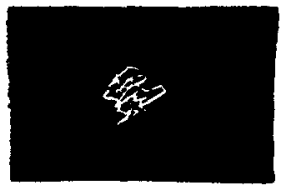

2

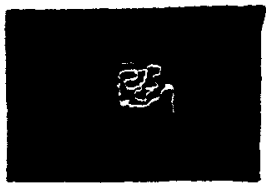

b.

a, b. Tracings made by Mr. Blasse in the "overhead register" steadiness test

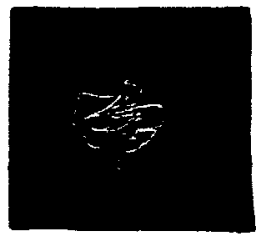

c.

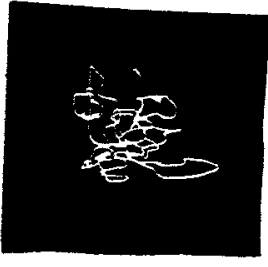

d.

c, d. Very good tracings made by other subjects in the "overhead register" test

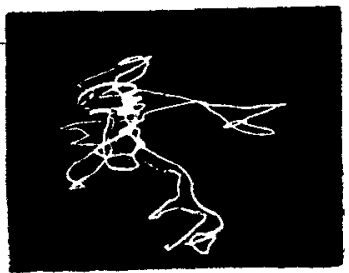

e

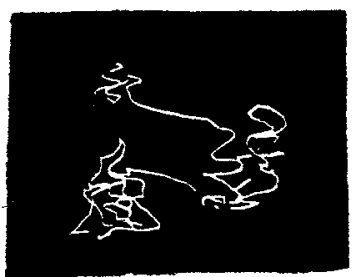

f.

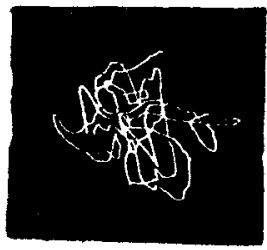

g.

e, f, g. Tracings made by subjects under suggestion. (e) suggestion of moving to nght, $(f)$ of moving to left and $(g)$ of being "awfully shaky" 
nounced than before. He was able to keep the pointer in a hole of $13 / 64$ inch diameter for several seconds without touching, while most of the other subjects incessantly bumped the sides of a hole twice as large.

A more realistic test consisted in arranging a pointer which played against a smoked surface two inches below the end of the barrel while the subject armed at a target and discharged a blank cartridge in the same manner employed in actual shooting. The accompanying records (Fig. II) tell the story better than words. It will be noted that the tracings made by $\mathrm{Mr}$. Blasse's gun cover an exceedingly small range, that the sight is quickly obtained and that immediately after the discharge the gun is moved perpendicularly upward with a quick pull. The records for the other subjects show a faltering over a large area, with wide excursions in all directions. As the gun wabbles about the subject attempts to pull the trigger when the bead happens to hit upon the bullseye, and in this effort he is doubtless often unsuccessful The clean cut, upward withdrawal of the rifle after firing is seldom shown, sometimes because it does not occur and often because the subject permits the gun to be jerked backward from its original position upon firing. It should be remembered that an excursion of a small fraction of an inch shown on the tracing would mean a wide miss when projected to a field 200 yards distant. Some of the subjects would probably have done well to "hit the side of a barn."

Aside from general steadiness and control of the bodily muscles, the action of the fingers controlling the trigger may have some bearing upon the result. Mr. Blasse habitually uses a sensitive trigger and in his opinion the action of the forefinger muscles with the required delicacy and at the right moment is an essential factor in the success of the shot. An exact graphic record of the control of these muscles was obtained by a simple apparatus. Over the bowl of a so-called "thistle tube" is stretched a delicate rubber membrane and the outlet at the small end of the tube is connected with a tambour. With the hand resting comfortably, the subject was given several tasks to perform with his forefinger. First he was asked to tap the membrane many times in succession, each time with equal force (see Fig III). In the figure, the height of the line gives a picture of the force exerted by each tap. It requires no special measurement to see Mr. Blasse possessed some superiority in this capacity.

Using the same apparatus, the subject was asked to press down with uniform pressure and speed upon the membrane, 
FIG. II

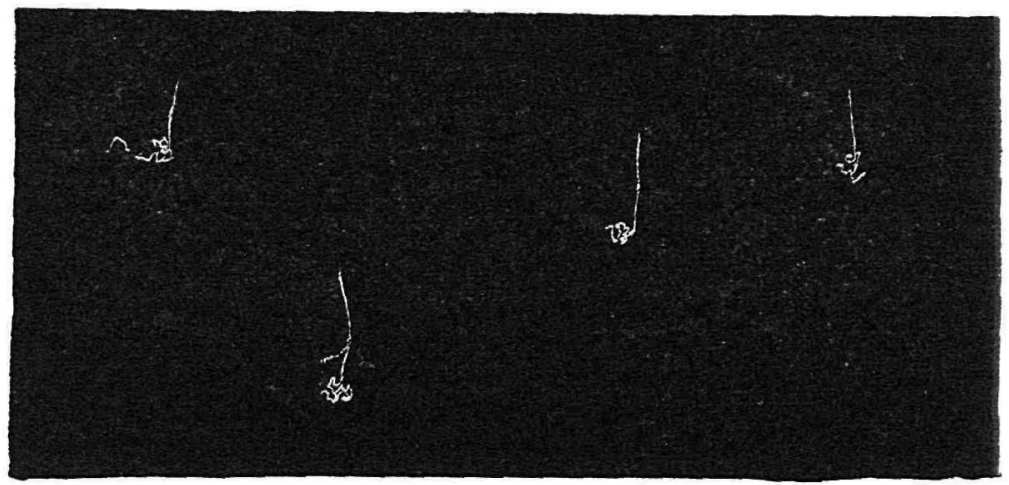

a.

a. Records made by Mr. Blasse, showing the movements of the end of the rifle during aiming and firing. The straight vertical lines are made by the quick "pull away" of the rifle immediately after firing.

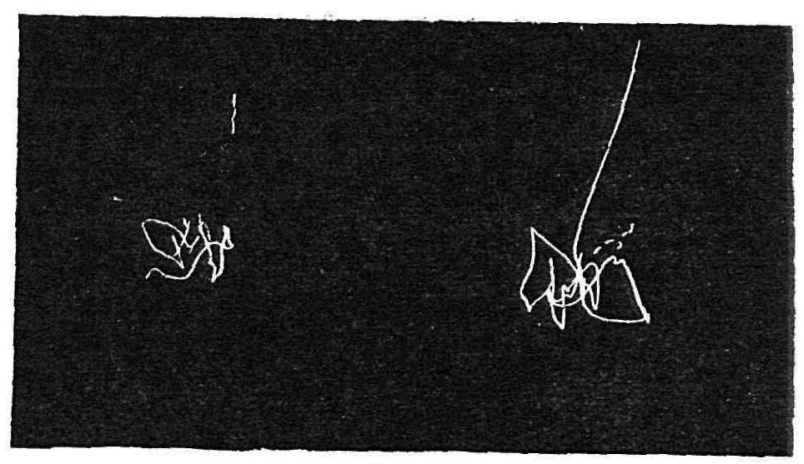

b.
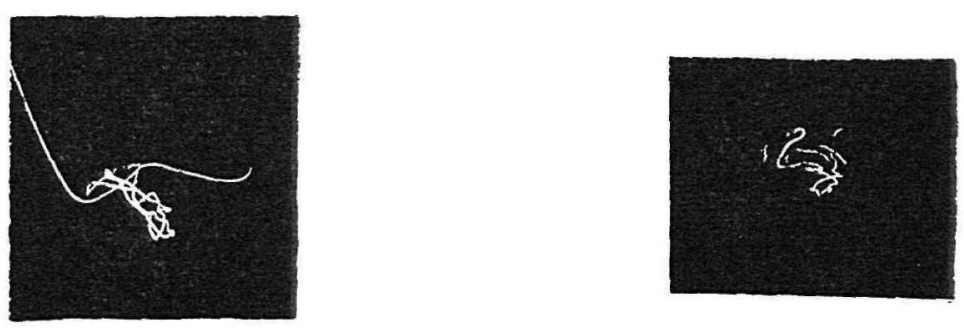

c.

d.

b, c, d. Aiming and firing records made by other subjects. d. was made by a very good rifleman. 
FIG. III

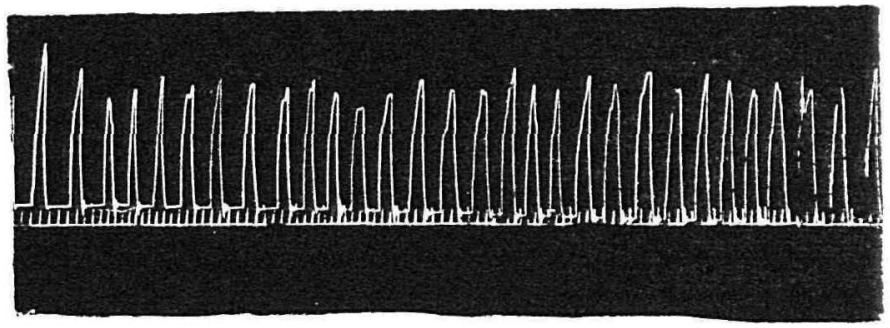

Record of Mr. Blasse attempting to tap the membrance of the thistle tube with uniform force. The height of the lines indicates the force of each stroke.

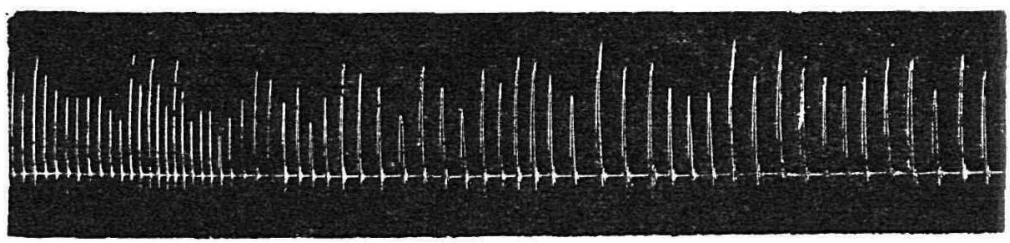

Record of an average subject in tapping.

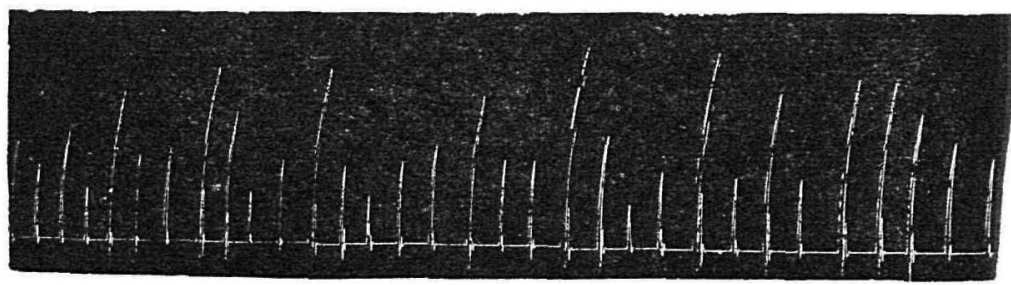

Record of a very poor subject. 
and then to release the pressure with equal speed. This test, which was repeated many times in succession, is a very trying test of muscular control. If speed and force remained constant throughout, a symmetrical curve would appear. The test was tried in several different positions, i.e., pressing downward perpendicularly, pressing it toward the left, pulling inward against it as one would in pulling a trigger. The curves illustrated here (Fig. IV), are for the latter task, and Mr. Blasse's superiority here is quite clear. The best record from the student subjects is not greatly inferior, however, and with practice this student might have developed to the marksman's equal in this respect. In general, however, Mr. Blasse not only greatly surpasses the ordinary person in steadiness of motor control, but also in the delicacy and precision of action with the trigger finger, which may, indeed, be of considerable significance

The last group of experiments include certain tests in reaction time, the Hipp chronoscope being employed. All of the subjects were given preliminary practice, followed by 50 trials in each test. In single reactions to sound (telegraph key) Mr. Blasse showed an average of $119 \sigma^{4}$ as compared to an average of $124 \sigma$ for 8 students, although three of these were superior to him. A similar test for a reaction to a sharp stroke of the left hand, gave an average time of $115 \sigma$ for Mr. Blasse, and an average of $118 \sigma$ for the 7 subjects. Again three students responded more quickly in this test than did the marksman.

Another test, employing the same general apparatus, has more significance for the present purpose. An apparatus was provided such that the subject reacted by pulling a trigger in the same manner as employed in firing a gun. About 10 feet in front of the subject was placed the white end of a metal rod about a quarter of an inch in diameter which showed plainly on a black ground. Fastened to the end of a metal rod which was blackened so as to be almost invisible against the ground was placed another white piece of metal about $1 / 6$ of an inch in diameter, which could be moved about in various ways and could at any time be brought into contact with the stationary spot of white first mentioned. When a contact was made between the two, the electric current was closed which set the chronoscope in action. The chronoscope was stopped when the subject pulled the trigger and thus the time between the coincidence of the spots and the pulling of the trigger was recorded. By moving the free spot of white all around the other, now approaching, now receding, now wavering here,

${ }^{4} \sigma$ equals a thousandth of a second 
FIG. IV

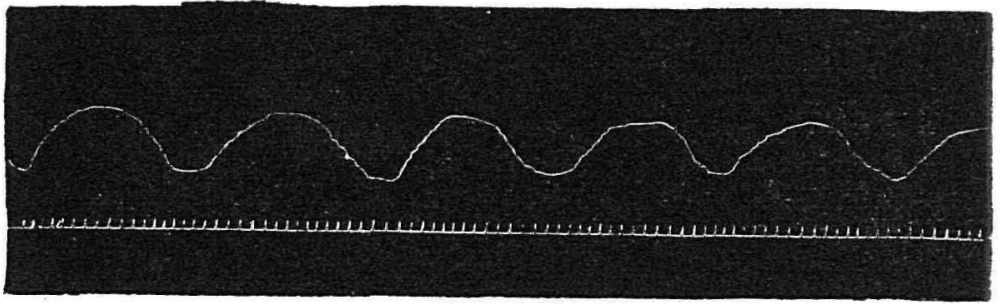

Record of Mr. Blasse attempting to pull in and release the membrane of the thistle tube with uniform force.

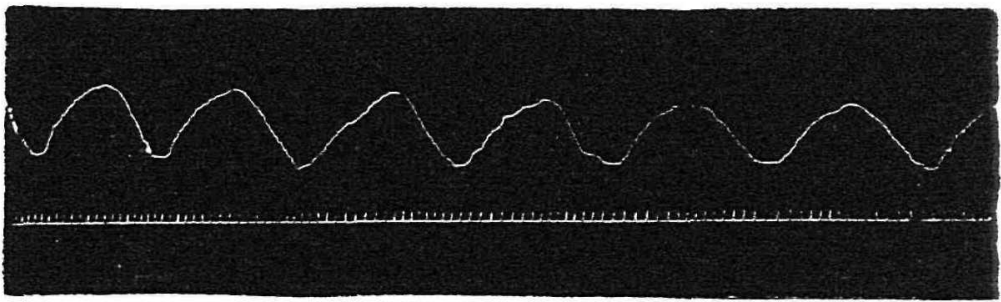

The best record among the other subjects with the thistle tube as above.

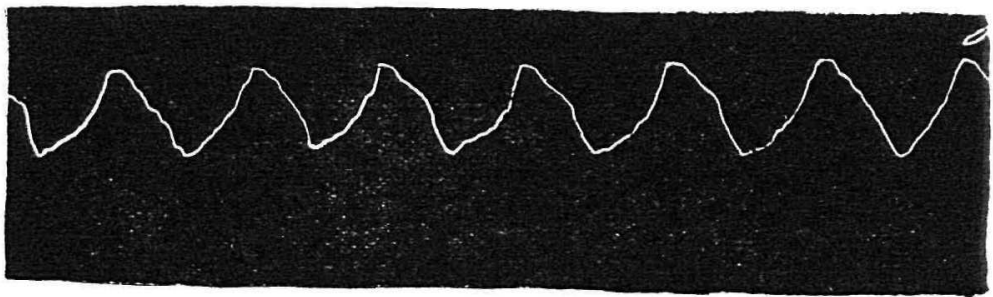

One of the poorer records. 
now there, before a contact was actually made, a situation similar to the bead of a rifle wavering about a target in actual shooting was secured. The speed and uniformity with which the trigger is pulled after the imitation sight coincides with the imitation target, gives a significant account of the coordination between eye and hand which would be of influence in shooting. If the trigger is pulled too quickly or too slowly, the bullet goes wide of tts mark. What will be of special significance here is not only the speed of the reaction but its uniformity during a series of shots. If, for example, after the impulse to pull the trigger is mitiated, the time elapsing before it is actually pulled is now $100 \sigma$, now $200 \sigma$, and at other times $150 \sigma, 250 \sigma$ or $90 \sigma$, it can readily be seen that the discharge will usually not occur at just the right time, since the gun is usually in motion. The impulse to pull, probably occurs just as, or just before, the bead begins a passage over the bullseye. But suppose the time elapsing between impulse and pull is always uniform, that is to say, for illustration, it is always $150 \%$ or but a few sigma more or less. Since the interval is always about the same, the shooter comes through experience, through trial and success, to know just when to start the impulse to fire. $\mathrm{He}$ would then meet with success, even if his reaction time were fairly slow, although of course, the speedier the action the better.

The results from the reaction time tests are presented in briefest form by giving the average time with the mean variation, (this figure being the more significant) together with the number of premature reactions, or cases in which the subject reacted before the two white spots touched, i.e., before the signal was given. Fifty trials were given each subject in each test.

The following table gives the results for the irregularly moving test.

Mr. Blasse

Av. sigma

158

M. V.

18

Premature reactions 0

198

Av of 8 other subjects. 96

Mr. Blasse's reactions are on the average much quicker, and very much more uniform, the $M V$. of the others, on the average, being 5 times as great. Moreover, Mr. Blasse did not, in a single instance, "jump the gun," while the others did so on the average of one time out of 9 trials.

The following figures give the results of tests in which the 
movable white spot instead of wavering about in an indefinite manner as above before finally touching the stationary spot, approached in one direction at a uniform speed.

Mr. Blasse-approaching from above downward

$\begin{array}{ccc}\text { Av. } & \text { M. V. } & \text { Premature reactions } \\ 155 & 10 & 0 \\ 162 & \text { Approaching from below. } & 0 \\ 13 & \\ 152 & \text { Approaching from right. } & 0 \\ 9 & \text { Approaching from left. } & 0 \\ 150 & 10 & 0\end{array}$

Av. of from 8 to 10 other subjects.

Approaching from above

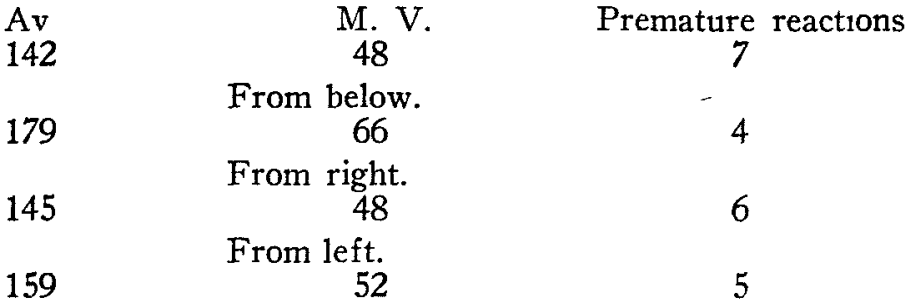

In these tests, Mr. Blasse shows no superiority as regards speed of reaction, but this is due in large measure to the fact that many reactions of the other subjects followed upon premature impulses although the chronoscope was started before the reactions were completed, thus accounting for such impossible reaction times as $50 \sigma$ etc., which cut down the average markedly In many cases, as shown above, the impulse was initiated so early that the reaction was completed before "sight" and "bullseye" came into contact In no case did Mr. Blasse make such a premature reaction. In fact the regularity of his responses is the most conspicuous superiority, and, as indicated earlier, this is a vital capacity in the development of marksmanship, since it offers the possibility (no matter what the actual time of reaction may be) of establishing a habit with regard to the moment of initiating the impulse to "pull" the trigger. So long as the reaction time is approximately the same, and the movements of the gun can be farrly well controlled, experience can readily supply the other requisite elements. 
We may now summarize briefly the capacities which seem to characterize the champion marksman. His vision is not exceptional, although he has no special eye defects. It seems reasonable to suppose that for practical shooting, exceptionally keen eyes are not essential and even such eye defects as myopia, astigmatism and the like (which may be corrected by proper glasses) are not prohibitive of good marksmanship. Muscular difficulties of convergence which interfere with the movements of the two eyes together need not be a handicap since many expert marksmen shoot with one eye closed.

Muscular steadiness of the members of the body which are brought into action in holding the gun on its mark is of great importance. This was shown by all the tests that were employed Of special import is the ability to resist the influence of various disturbing factors upon one's steadiness, such as slight distractions and especially subjective anxiety and autosuggestion. The type of indrvidual who is keenly aware of and disturbed by his own unsteadiness during shooting only increases his unsteadiness thereby. While muscular steadiness is doubtless not absolutely essential to skill in shooting, as evidenced by several good marksmen who are quite shaky, it certainly affords a great advantage.

A nicety of muscular control of the fingers involved in manipulating the trigger perhaps plays a rôlealthough this function should really be considered in connection with the results of the tests on reaction time. Our expert marksman showed a remarkably fine correlation of eye and hand in these tests. While nerved up, in such a test and likewise in actual shooting, to a high sensitivity, "breaks" in nervous control did not occur. The ordinary shooter, like the nervous athlete waiting the pistol, often "jumps the gun," that is, he pulls the trigger before the sight is perfect, or if he does not do this, he is unprepared when the proper moment comes, and the trigger is pulled too slowly. But our expert fails in neither of these respects. His reaction is never premature, and when bead and target are in the proper relation, the reaction is made with dispatch and always with great uniformity. This calm, speedy and uniform reaction is of utmost importance. Coupled with steadiness of general muscular control, this capacity seems to be essential to skill in marksmanship.

To what extent is the superiority of the expert in these tests due to training in shooting and to what extent is it due to native ability-ability possessed before practice,-is a matter of importance upon which our present data afford little evidence. If we pick out a hundred men at random-say, any hundred of 
the men conscripted for government service-we would find that a few had received considerable, many a little and many no previous practice with a rifle If we wished to select from these, a number who are destined after practice, to become the best sharpshooters, we must employ tests that divulge native, and not acquired ability. It must be admitted that much more work must be done with these and other tests before this matter can be decided with certainty. It will be necessary to test individuals who have had much experience in shooting but have developed but little skill. If our tests are valid for this purpose, such individuals would do poorly in them. It may be stated, at least, that of the 10 subjects beside Mr. Blasse who went through the tests, one whose experience in shooting was considerable was among the poorest in the tests, while one who was the best in most of the tests had shot a gun less than a dozen times during his life.

It would be interesting to know, also, what influence various habits of life have upon the capacities required for marksmanship. It might be said in passing that Mr. Blasse is a man of good habits. He has never indulged in smoking or drinking. With regard to smoking, data obtained in these tests from one rather heavy smoker are interesting. In the steadiness tests he was poorer than any of the other subjects and his shakiness was particularly noticeable just after smoking a cigarette. If permitted to wait for 12 or 15 minutes, his steadiness returned to his norm, however. This single record cannot be taken to prove that smoking makes one decidedly unsteady, for many other factors must be considered and many other similar tests performed to make certainty of such a statement. The fact may be that the person who is naturally unsteady or nervous, may more readily become addicted to smoking That is, some people may smoke because they are "highly strung" or nervous, and are not so unsteady just because they smoke.

This examination of skill in marksmanship is, of course, preliminary and uncertain. Some of the tests here described could well be omitted for practical purposes, and doubtless all could be improved and new ones should be added. Some may say, in fact, "Why not give a man a gun and let him show his ability by shooting a few times!" Well, there are good reasons. In actual shooting, experience plays too large a rôle. The novice must be shown how to hold the gun, whether to grasp it tightly or laxly, whether his bodily muscles should be held lax or taut, whether he should pull the trigger slowly or with a sudden jerk, how much of the front sight should be visible in the notch of the rear, whether he should aim at the bottom, center or top 
of the bullseye, whether he should "hold steady" on the bullseye or move up to it, not to mention the adjustment to and fear of the noise, flash and "kick" The knowledge of these conditions and the adjustment to them depends mostly on experience in shooting, not upon innate ability to eventually become a good marksman The laboratory tests aim to measure this undeveloped ability, although the present series may not do so adequately. 